\title{
Automatisation du contrôle radiologique de l'air des locaux de travail de Valduc
}

\author{
J.Y. HERVE, L. CAMUS, J.C. LEROUX*
}

(Manuscrit reçu le 10 janvier 1991)

RÉSUMÉ Les installations du Centre de Valduc requièrent un contrôle radiologique de l'air des différents locaux à risque radiologique. Pour cela, les agents de radioprotection déposent quotidiennement 230 filtres sur des têtes de prélèvement à proximité des postes de travail. Pour mesurer la radioactivité éventuelle retenue sur les filtres, le Service de protection contre les rayonnements de Valduc met en cuvre un système automatisé qui permet de reconnaître l'origine des filtres, d'en effectuer les mesures et de mettre en forme les résultats obtenus.

Les filtres sont identifiés par des étiquettes à codes à barres lues par un lecteur optique implanté dans chacune des deux chaînes de mesure. Ces dernières sont des ensembles de comptage de faible activité $\alpha$ et B de type Nu-16 (Numelec Ins.) équipés de compteurs à circulation fonctionnant en régime proportionnel. Le pilotage de l'ensemble et la centralisation des données sont assurés par un microordinateur de type IBM-PC. Différentes éditions des résultats peuvent être obtenues : résultats quotidiens, mensuels, trimestriels ou annuels.

Le mode de fonctionnement d'un tel système est satisfaisant ; il a permis de dégager une charge de travail équivalente à un agent.

ABSTRACT The facilities of the "Centre d'études de Valduc" require air control in the different rooms where a radiological hazard exists. To this purpose, every day, the radiation protection technicians put $\mathbf{2 3 0}$ filters on the sampling heads in the working places proximity. To measure any radioactivity retained by the filters, the Valduc Radiation protection service uses an automated system, which identifies the filter origin, carries out the measurements and produces the results.

Filters are identified by bar code labels, which are read by an optical reader located in each of the two measurement lines. These are Nu-16 (Numelec Ins.) type low $\alpha$ - and $B$-activity counting sets, equipped with proportional mode circulation counters. An IBM-PC type microcomputer is used for the whole assembly monitoring and the data centralization. Results can be edited in different forms : daily, monthly, trimestrial or annual results.

The system operating mode is satisfying, and has allowed to clear a one-man equivalent work-load.

\footnotetext{
* Commissariat à l'énergie atomique, Centre d'études de Valduc, Service de protection contre les rayonnements, 21120 is-sur-Tille.
} 


\section{INTRODUCTION}

La réglementation concernant la protection des travailleurs contre les rayonnements ionisants [1] prévoit que tout exploitant de zone contrôlée effectue les contrôles de la contamination radiologique atmosphérique des lieux de travail. A Valduc, ces contrôles sont effectués au moyen de filtres déposés sur des têtes de prélèvement dans les locaux de travail de la zone contrôlée. Pour cela, les équipes de radioprotection déposent et retirent quotidiennement 230 filtres et 70 filtres de façon hebdomadaire. La présence éventuelle de radioactivité retenue sur les filtres est déterminée ultérieurement au moyen de bancs de comptage.

Afin d'optimiser la charge de travail correspondante, le Service de protection contre les rayonnements (SPR) de Valduc a mis en œuvre, récemment, un système automatisé qui permet de reconnaître l'origine des filtres, d'effectuer les mesures et de mettre en forme les résultats obtenus. Ce système a été réalisé par la société Numelec Ins. à partir d'un cahier des charges écrit par le SPR.

L'exposé qui suit décrit l'architecture du système et les résultats obtenus après 6 mois d'exploitation et le gain de productivité qui en résulte.

\section{DESCRIPTION DU SYSTĖME}

\section{Repérage des filtres}

Les équipes responsables de la radioprotection dans les bâtiments du centre sont chargées de la mise en place et du retrait des filtres selon la répartition donnée dans le tableau I. Ces filtres (SOFILTRA C 577), d'un diamètre de $75 \mathrm{~mm}$ et $130 \mathrm{~mm}$, sont disposés sur des têtes de prélèvement présentant un débit commun d'aspiration de 1 à $3 \mathrm{~m}^{3} / \mathrm{h}$.

Les filtres sont identifiés par des numéros à 8 chiffres imprimés en codes à barres sur des étiquettes collées directement sur leur surface (fig. 1). Le standard de codes à barres utilisé est du type "2 parmi 5 entrelacé" sans digit de contrôle.

L'identification utilisée est la suivante :

- 2 chiffres : numéro du bâtiment ;

- 2 chiffres : numéro de la tête de prélèvement dans le bâtiment considéré ;

- 3 chiffres : date de mise en place (numéro du jour dans l'année) ;

- 1 chiffre : numéro de l'année $(9=1989 ; 0=1990 ; 1=1991$; etc.).

Cette prestation est assurée par une société qui nous fournit chaque mois les filtres étiquetés avec les codes à barres et conditionnés par bâtiment et par jour 
TABLEAU I

Implantations et caractéristiques des filtres de contrôle atmosphérique

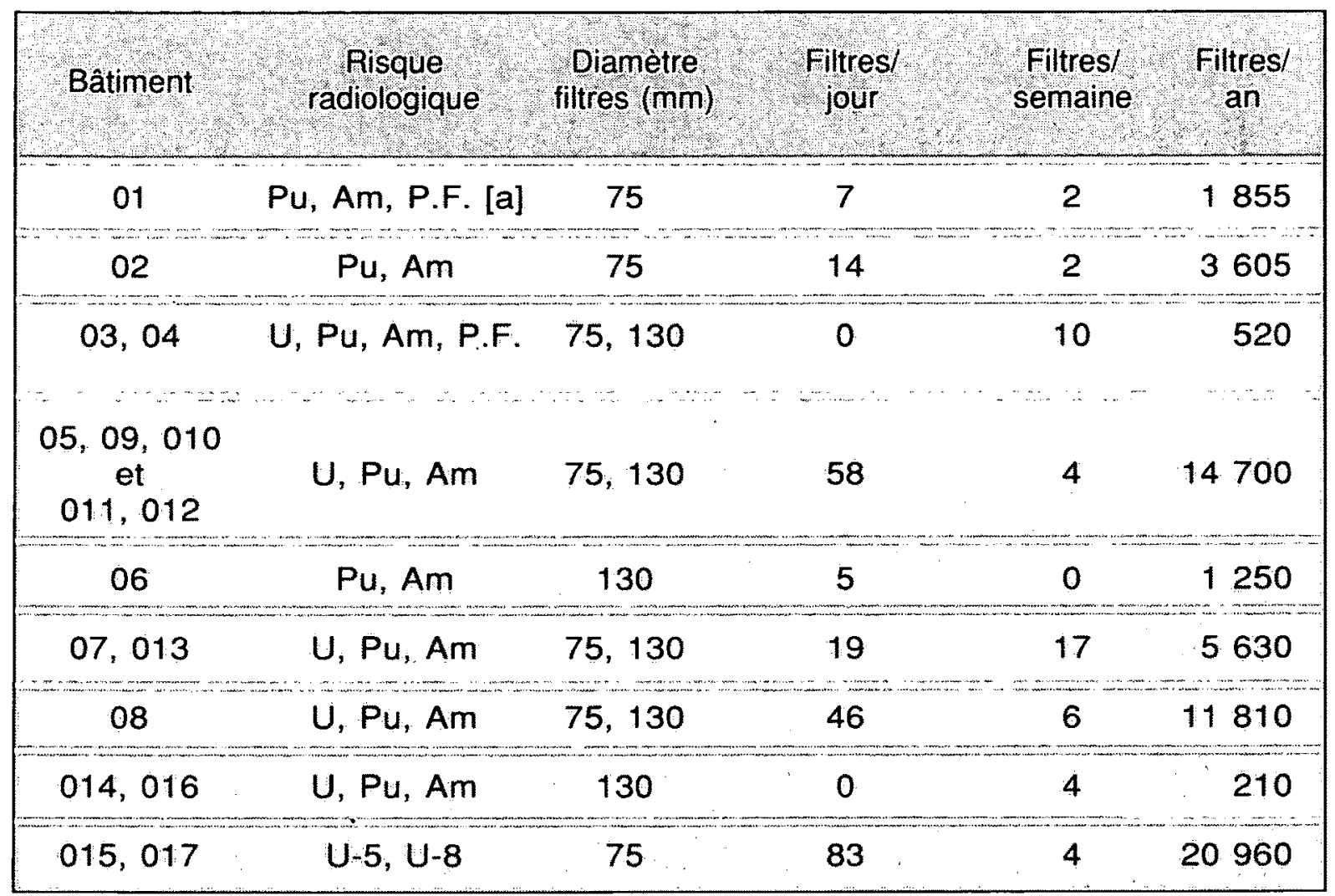

[a] : P.F. : produits de fission non identifiés.

\section{Appareils de comptage}

Les deux appareils utilisés sont des ensembles de comptage de faible activité $\alpha$ et $B$ type Nu-16 (Numelec Ins.) équipés de compteurs à circulation fonctionnant en régime proportionnel (type $S 34$; diamètre $130 \mathrm{~mm}$ ). Ces appareils sont dotés de passeurs d'échantillons capables de recevoir 50 filtres chacun ; ces filtres sont comptés pendant $1 \mathrm{~min}$.

Les seuils de détection calculés d'après [2] sont donnés sur le tableau II pour différents débits d'aspiration et pour un même temps de prélèvement de $25 \mathrm{~h}$. L'activité minimale détectable sur un filtre, pour un temps de comptage d'une minute, un débit d'aspiration minimal de $1 \mathrm{~m}^{3} / \mathrm{h}$ et un temps de prélèvement de $25 \mathrm{~h}$ est de $0,8 \mathrm{~Bq}$ en $\alpha$ total. L'activité volumique calculée correspondante est de 0,4 LDCA pour le plutonium (limite dérivée de concentration dans l'air pour une exposition professionnelle de $2000 \mathrm{~h} / \mathrm{an}$ ). Tous les résultats inférieurs ou égaux à cette valeur sont dits "non significatifs" (NS), d'un point de vue radioprotection. Afin d'uniformiser les éditions des résultats, nous avons choisi d'adopter ce seuil pour tous les radionucléides susceptibles d'être rencontrés dans nos installations. 


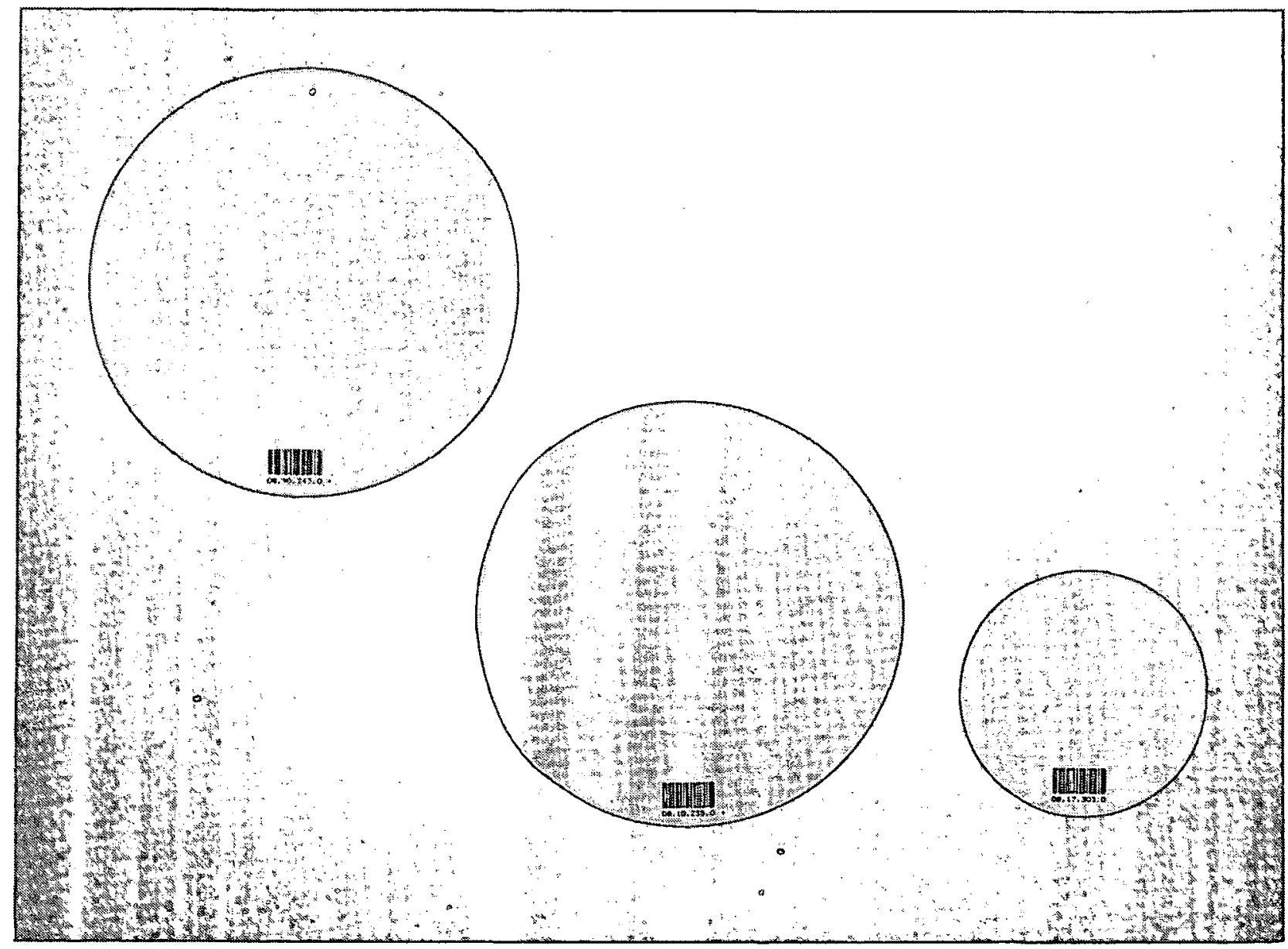

Fig. 1. - Repérage par étiquettes à codes à barrres des filtres

Les filtres présentant une activité $\alpha$ supérieure à ce seuil de 0,4 LDCA subissent, de plus, une décroissance de 4 jours, afin de s'affranchir de l'activité due aux descendants solides du radon déposés sur les filtres, et retournent dans la chaîne de comptage. Ce temps de décroissance appliqué pour les filtres positifs peut paraître trop court, mais il est le résultat d'un compromis entre la précision de la mesure et les impératifs d'exploitation des installations pour lesquelles il convient de fournir des résultats le plus rapidement possible.

\section{Saisie de l'identité des filltres}

Les étiquettes sont lues par un lecteur optique à barrettes CCD linéaire de type LEMCO 7 implanté dans chaque appareil de mesure (fig. 2). Le lecteur est connecté à l'électronique $\mathrm{Nu}-16$ et au centralisateur via un décodeur par des liaisons RS 232C. La distance de lecture entre l'étiquette à codes à barres et le capteur est de $10 \mathrm{~mm}$; il n'y a pas de sens de lecture privilégié, l'appareil lit les codes durant le premier passage et lors du retrait, si la première lecture ne s'est pas effectuée. 
TABLEAU ॥

Seuil de détection des $\mathrm{Nu}-16$

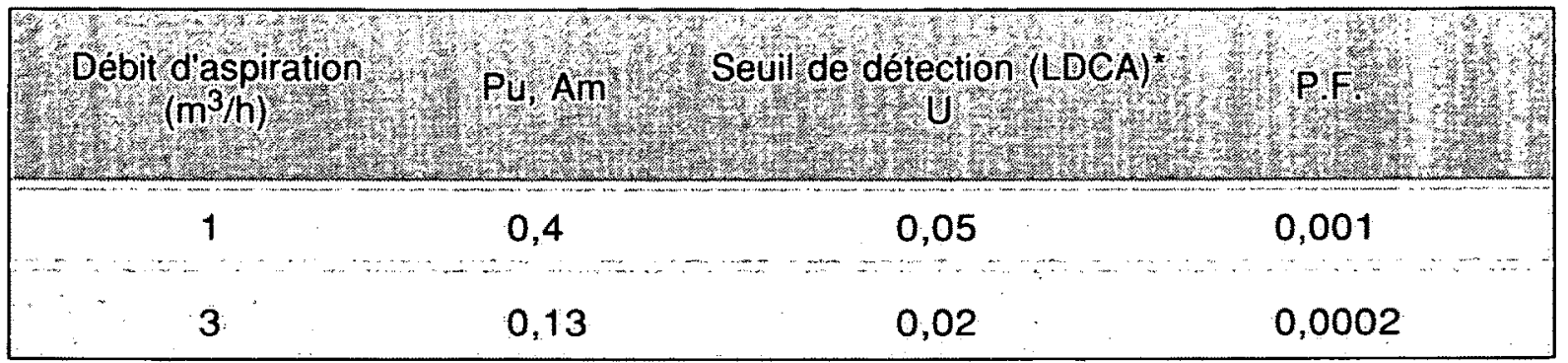

* LDCA : limite dérivée de concentration des radionucléides dans l'air pour une exposition professionnelle de $2000 \mathrm{~h} / \mathrm{an}$ [2].

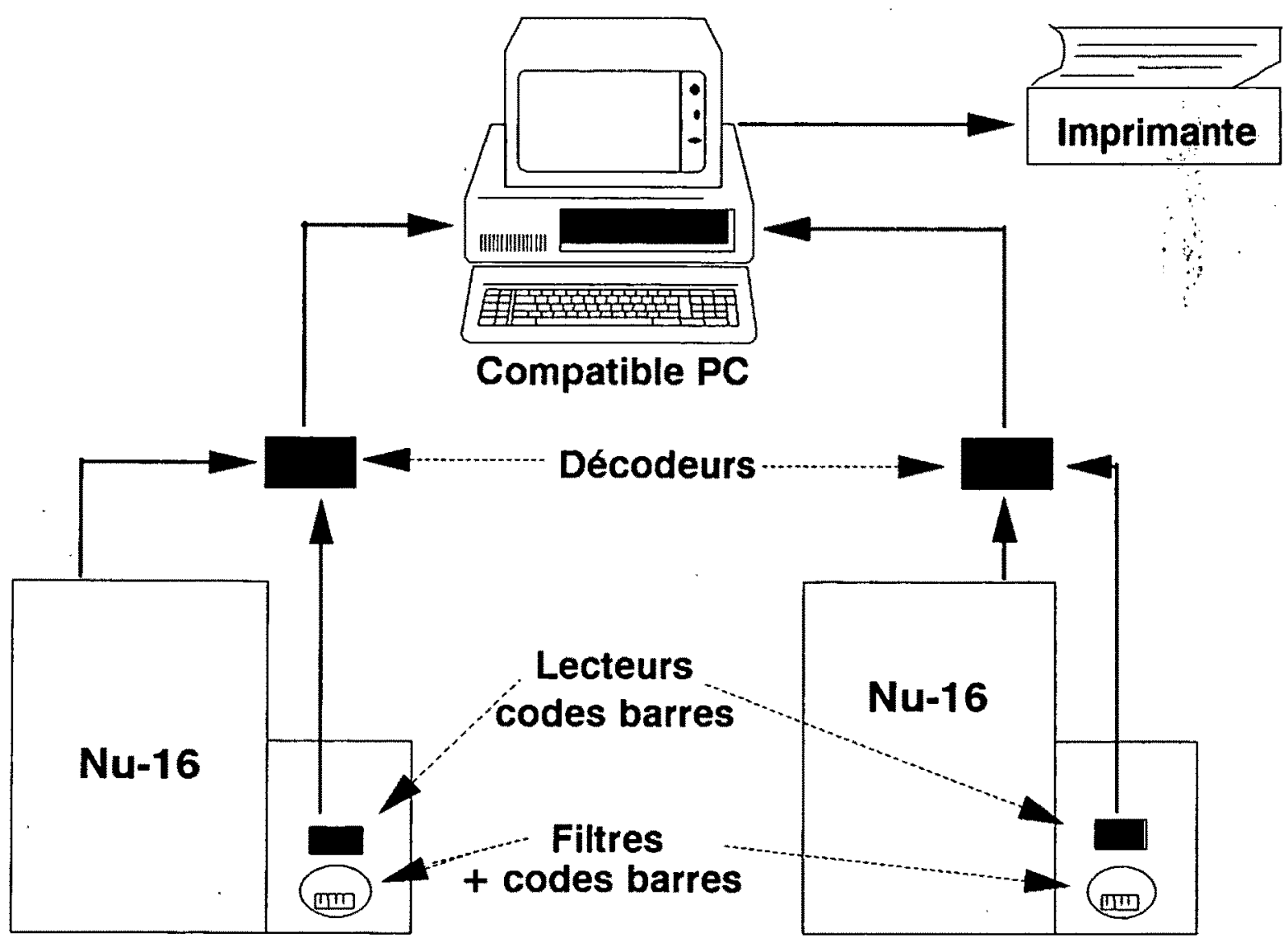

Fig. 2. - Configuration du système 


\section{Centralisation des données}

Le centralisateur est constitué d'un microordinateur de type IBM-PC équipé d'un disque dur de $20 \mathrm{Mo}$, d'une carte multivoies à liaison série RS $232 \mathrm{C}$ et d'un système de sauvegarde des résultats (streamer + cartouches de $40 \mathrm{Mo}$ ).

Le logiciel du centralisateur comprend :

- un système d'exploitation MS-DOS avec ses commandes standards ;

- le pilote et le programme de test de la carte multivoies ;

- le logiciel centralisateur qui assure la visualisation des résultats de comptage en cours d'acquisition ou des résultats acquis précédemment ;

- un ensemble de programmes spécifiques sous progiciel "Open Access" réalisant les fonctions d'édition et de traitement qui seront décrites par la suite.

La base de données comprend principalement :

- les caractéristiques complètes des têtes de prélèvement (fichier maître) ;

- les résultats définitifs et temporaires; nage.

- la liste descriptive des sources étalons et les résultats d'étalon-

\section{Traitements, éditions}

\subsection{Mesures instantanées et après décroissance}

Après chaque mesure, pour l'ensemble d'un passeur d'échantillons traité, le programme imprime :

- les résultats de mesure de bruit de fond et d'étalonnage ;

- une liste des mesures de filtres dont le comptage s'est effectué ;

- une liste des filtres positifs qu'il convient de mettre en décroissance pour comptage ultérieur ;

- un message d'erreur pour les séquences mal effectuées.

Les résultats de comptages négatifs, et positifs après décroissance, sont inclus dans les fichiers définitifs (par opposition aux résultats de comptage positifs, avant décroissance, dont les valeurs sont transférées dans un fichier temporaire). 


\subsection{Editions}

Le programme d'édition est lancé manuellement par l'opérateur, il comprend :

- l'édition des résultats de filtres déclarés définitivement positifs, classés par bâtiment et par jour (toutes les données sont calculées en fraction de LDCA) ;

- l'édition des résultats mensuels par bâtiment ;

- l'édition des résultats trimestriels et annuels par bâtiment.

Une liste des résultats d'étalonnage peut être également imprimée, elle contient la date, l'heure, les valeurs de bruit de fond et le taux de comptage de la source de contrôle.

\subsection{Archivage}

Une sauvegarde mensuelle de tous les résultats est effectuée sur disquette. La capacité du disque dur permet de stocker les résultats complets d'une année. Une sauvegarde annuelle est effectuée sur le streamer, les résultats de l'année écoulée sont alors détruits.

Toutes les coupures de secteur sont traitées par le système qui, bien qu'équipé d'un onduleur, distingue 3 défauts d'alimentation en cas de défaillance de ce dernier :

- défaut de l'appareil de comptage ;

- défaut de l'ordinateur seul ;

- défaut simultané (appareil de comptage + ordinateur).

\section{RÉSULTATS}

\section{Tableaux de résultats}

\subsection{Résultats mensuels}

Un extrait de résultats obtenus dans le bâtiment de retraitement des déchets radioactifs du Centre, durant le premier mois d'exploitation du système, est présenté dans le tableau III. Ce type de tableau, généré par le programme du système et diffusé mensuellement dans chaque bâtiment, permet au responsable de la radioprotection ainsi qu'au chef de l'installation concernée de juger au premier coup d'œil de l'état radiologique de l'air des différents locaux dont ils ont la charge.

On peut ainsi relever dans ce cas présent, et à partir du tableau complet, que l'ensemble des filtres ne présentaient que des valeurs non significatives. Les valeurs observées pour les deux autres mois d'utilisation sont similaires. 


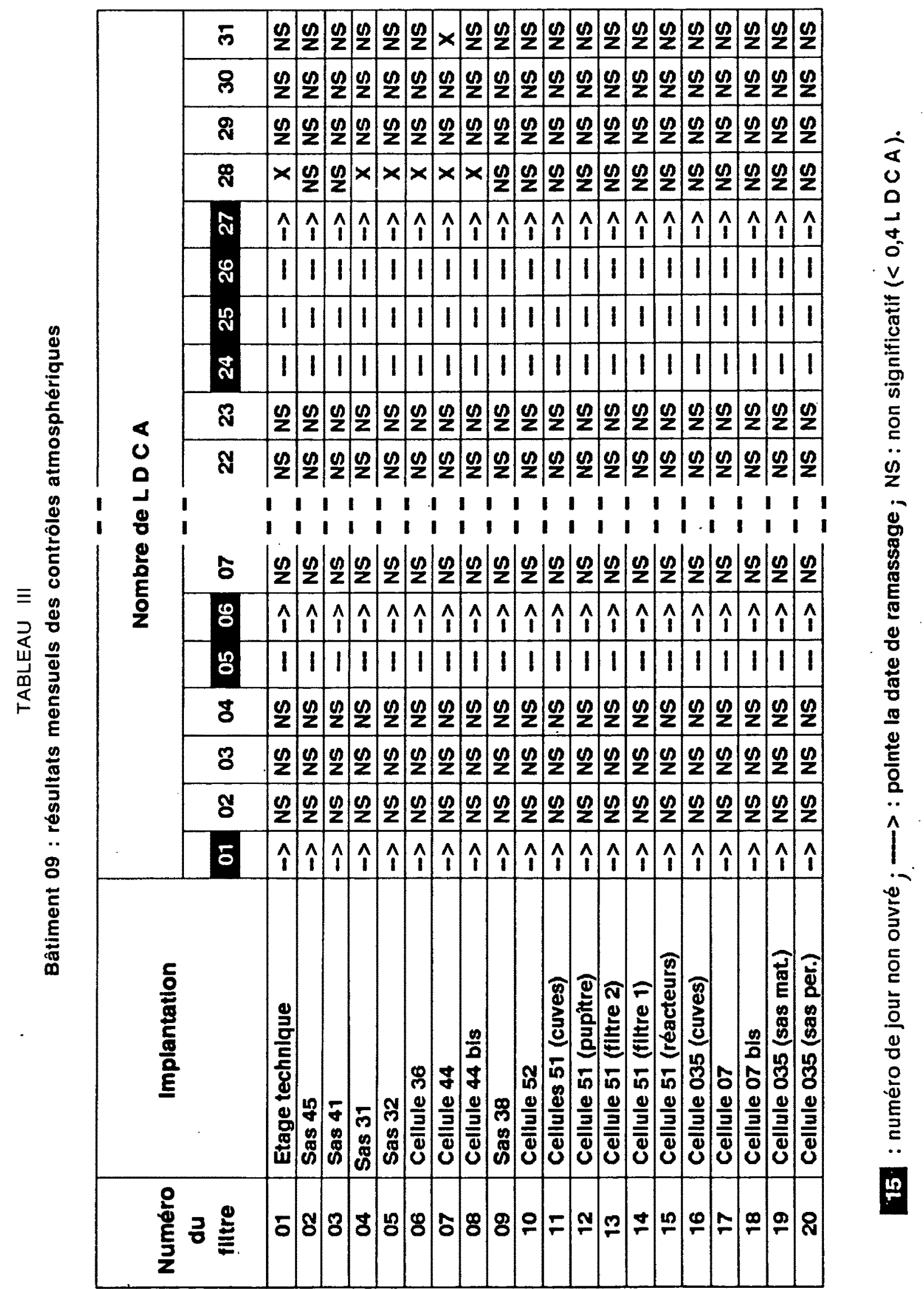




\subsection{Résultats trimestriels}

Le tableau IV, qui peut être généré à la demande par le programme du système, présente une synthèse des résultats obtenus durant la période trimestrielle de mise en exploitation. On peut ainsi voir que, sur les 10173 filtres exploités, seuls deux présentaient des valeurs supérieures à la LDCA. Ces deux anomalies correspondaient à une perte réelle de confinement sur le filtre du circuit de ventilation d'une installation.

On peut, par ailleurs, mentionner que durant ces opérations seuls 10 filtres n'ont pu être identifiés par le lecteur ; 7 d'entres eux présentaient des étiquettes à codes à barres légèrement décollées, les 3 autres avaient été mal positionnés dans le panier de comptage. Dans ces cas là, il convient d'introduire directement au clavier les codes mentionnés sur les étiquettes des filtres non reconnus.

\section{Gain de productivité}

L'exploitation du système, avant son automatisation, supposait :

- le repérage manuel au stylo et la gestion des filtres par les agents des équipes de radioprotection ;

- l'exploitation des machines de comptage et le report manuscrit des résultats à l'état brut des résultats sur un cahier.

Les temps de travail consacrés à cette activité, avant et après son informatisation, se décrivent comme indiqué dans le tableau V. Globalement, il en ressort que la mise en cuvre de ce système a permis de diminuer d'un agent l'effectif affecté à l'exécution de ces tâches.

Si l'on suppose que la durée de vie d'un tel système est de 10 ans et si l'on prend en compte la prestation correspondant à l'impression des étiquettes à codes à barres sur les filtres, la dépense annuelle est estimée à :

- amortissement de l'appareillage $25 \mathrm{kF}$

- étiquettes à codes à barres $140 \mathrm{kF}$

TOTAL $165 \mathrm{kF}$

Cette dépense, comparée au coût moyen annuel d'un agent d'éxécution, qui est de l'ordre de $335 \mathrm{kF}$ par an (référence 1990), permet donc de réaliser, pour cette activité, une économie annuelle de $170 \mathrm{kF}$.

Les potentialités du logiciel de traitement permettent, en plus, de faire un certain nombre de traitements (états mensuels, trimestriels et annuels) qui, outre la fonction d'alerte en cas de mesures significatives, n'étaient pas effectués auparavant. 
J.Y. HERVE

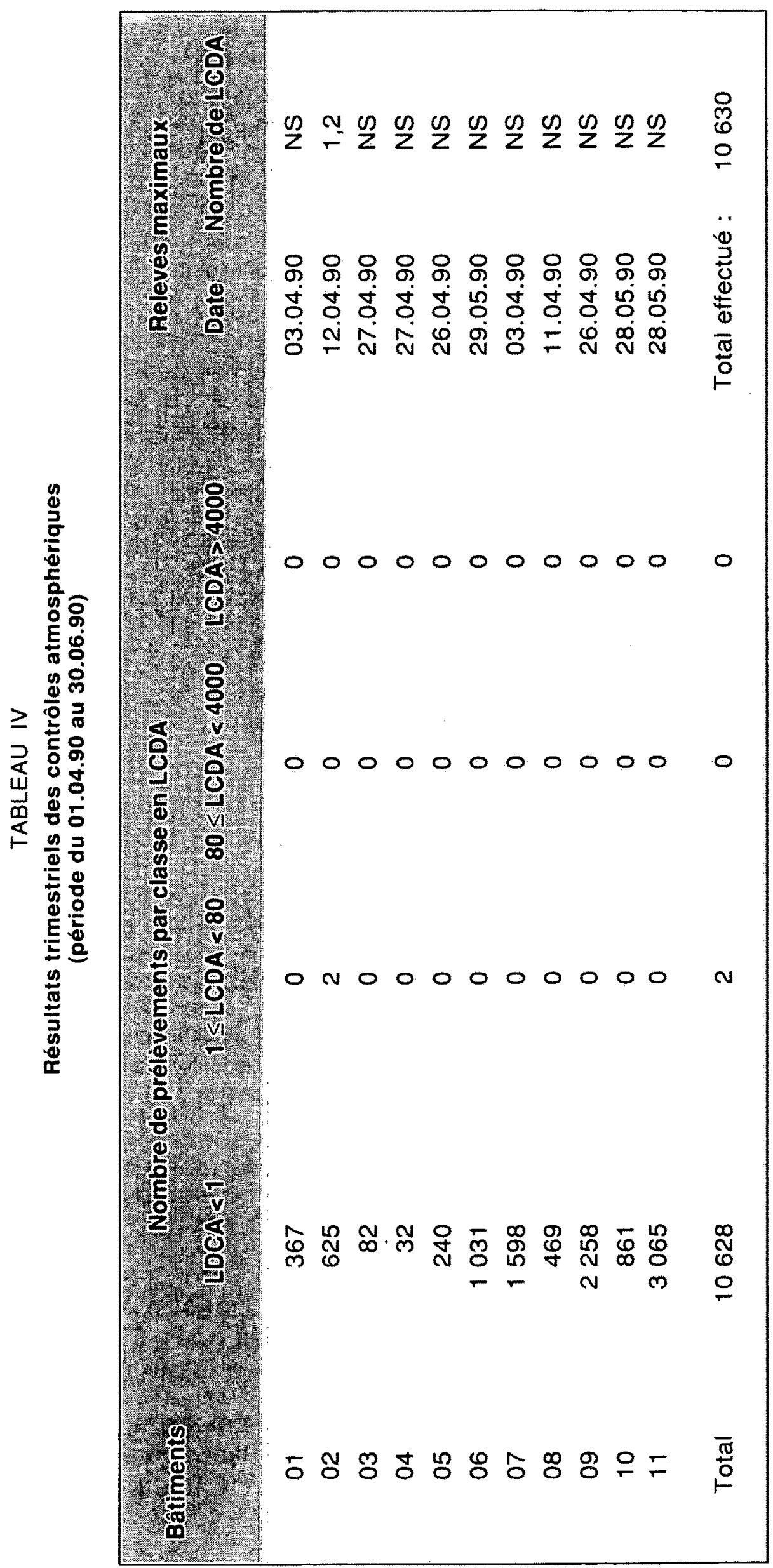


TABLEAU $V$

Effectif affecté au contrôle de la contamination atmosphérique

\begin{tabular}{|lcc|}
\hline & Avant automatisation & Apres automatisation \\
\hline $\begin{array}{l}\text { Nombre d'agents affectés } \\
\text { au repérage des filtres }\end{array}$ & 0,25 & 0 \\
$\begin{array}{l}\text { Nombre d'agents affectés à } \\
\text { l'exploitation des mesures et } \\
\text { à la transcription des résultats }\end{array}$ & 1 & 0,5 \\
\hline
\end{tabular}

\section{CONCLUSION}

Après six mois d'exploitation, on peut dire que le mode de fonctionnement d'un tel système est satisfaisant :

- l'utilisation d'étiquettes à codes à barres, comme mode de repérage des filtres, présente un bon niveau de fiabilité ;

- l'informatisation de l'ensemble de comptage permet aux équipes de radioprotection d'exploiter des résultats qui, avant la mise en œuvre de ce système, sommeillaient à l'état brut dans un cahier ;

- il a permis de dégager une charge de travail équivalente à 0,75 agent, soit 1 agent effectif.

Pour poursuivre l'optimisation de cette activité, une étude fonctionnelle est actuellement en cours afin que les résultats de comptage puissent être accessibles de manière quotidienne par les équipes de radioprotection à partir du réseau informatique ETHERNET dont est équipé le Centre de Valduc.

\section{RÉFÉRENCES}

[1] FRANCE. Affaires sociales et emploi (Ministère). - Décret no 86-1103 du 2 octobre 1986 relatif à la protection des travailleurs contre les dangers des rayonnements ionisants. JORF, 12 octobre 1986, 12295-12335 (Textes d'intérêt général 86-866) et décret $n^{\circ}$ 88-521 du 18 avril 1988 modifiant le décret $n^{\circ} 66-450$ du 20 juin 1966 relatif aux principes de protection généraux contre les rayonnements ionisants JORF, 6 mai 1988, 6224-6298 (Fascicule spécial 88-33).

[2] Commissariat à l'Energie Atomique. - Limite de détection d'un signal dans un bruit de fond. Applications aux mesures de radioactivité par comptage. Rapport CEA-R$5201,1983$. 